\title{
Rectus Abdominis Muscle
}

National Cancer Institute

\section{Source}

National Cancer Institute. Rectus Abdominis Muscle. NCI Thesaurus. Code C33449.

Two vertically parallel muscles located on the anterior abdominal wall, which originate on

the pubic crest and insert on the coastal cartilages of ribs five through seven and the xyphoid process; both muscles function to flex the lumbar spine. 